\title{
Categorization of the context within the medical domain
}

\author{
Hicham Ajami ${ }^{1}$, Hamid Mcheick ${ }^{1}$, Lokman Saleh², Rania Taleb ${ }^{1}$ \\ ${ }^{1}$ Université du Québec à Chicoutimi, Computer Science Department. 555, Boul De \\ l’Université, Chicoutimi, Quebec, G7H 2B1, Canada \\ ${ }^{2}$ Université du Québec à Montréal, Computer Science Department, Montréal. Case postale \\ 8888, Succursale Centre-ville, Montréal (Québec) H3C 3P8, Canada, \\ $\{$ Hicham.Ajami1, Hamid_Mcheick, Rania.Taleb1\}@uqac.ca \\ \{ Saleh.Lokman@courrier.\}uqam.ca
}

\begin{abstract}
The context itself has multiple meanings may vary according to the domain of application. This contextual flexibility was behind the emergence of so such huge number of context definitions. Nevertheless, all the proposed definitions do not provide solid ground for systems developers' expectations, especially in healthcare domain [1]. This issue prompted researchers to divide the context into a set of concepts that would facilitate organizing of contextual knowledge. The conventional taxonomies of context are always too complex, and we need to fight to make them useful in the intended application area. In this paper, we propose a new context classification which covers almost all the context aspects that we may need to develop a tele-monitoring system for chronic disease management.
\end{abstract}

Keywords: healthcare; pervasive computing; context categorization; medical context.

\section{Introduction}

The development of healthcare system should be supported by adequate context knowledge. Context and knowledge management is a very important feature to deliver the right service to the right person at the right moment. The conventional taxonomies of context are always too complex, and we need to fight to make them useful in the intended application area. Often, researchers have identified very general taxonomies of context to be used in different domains. However, these taxonomies cannot be applied a in specific domain [2]. Our objective in this paper is to present categorization of context in the medical domain. Such taxonomy aims to represent knowledge that can influence patient status, ensuring access to services and providing necessary monitoring. Although there are a plenty of context structures developed with different approaches, no agreed structure exists can be broadly used for developing telemedicine applications. This work is a new essay to make such structure more consistent. The remainder of this document is organized into three 
parts. In the first part we present the existing categorizations of the context while the second part describes our perception for medical concepts. The third part provides a case study to design healthcare system for Chronic Obstructive Pulmonary Disease (COPD) patients. Finally, we conclude this research work and give perspectives.

\section{State of the arts}

Although everyone has a general idea of what the context is, but it is certainly not easy to find a precise definition of context. There is a lack of standard definitions for this term. However, some common features can be extracted. Rey et al. [2] investigate these common characteristics which are discussed and agreed upon by the researchers in the contextual computing. (1) Context does not exist out of context, (2) Context is an information space that serves interpretation and (3) Context evolves, is structured, and shared. The existing work in the field of contextual management in ubiquitous healthcare environment suffers from absence of a detailed description of all dimensions of context. To make more meaningful contribution in this research area we need a good understanding of what are the most important aspects of context in the health care domain. The table below illustrates the most remarkable categorizations from 1994 to 2016.

Table 1. Dimensions of context.

\begin{tabular}{ll}
\hline Authors & Dimensions \\
\hline Schilit et al. (1994) [6] & Where, who, what \\
Ryan et al. (1997) [7] & Identity, time, location and environment \\
Hull et al. (1997) [8] & $\begin{array}{l}\text { User identity, locations, vital signs, air quality, network } \\
\text { availability }\end{array}$ \\
Pasco et al. (1998) [9] & Physical and logical \\
Franklin et al. (1998) [10] & Person's actions \\
Abowd et al. (1999) [11] & Primary and secondary context \\
Chen and Kotz (2000) [12] & Computing, physical, time and user \\
Petrelli et al. (2000) [13] & The material context and the social context \\
Gwizdka, (2000) [14] & The internal context and the external context \\
Klemke et al. (2000) [15] & Organizational, domain content based, personal and physical \\
Gross and Specht (2001) [16] & Location, Identity, Time, Environment or activity \\
Hofer et al. (2002) [17] & Physical and logical \\
Antti Aaltonen (2002) [18] & location, target, calendar, address book, users nearby, history, \\
& profile, direction and speed \\
Henricksen (2003) [19] & Sensed, Static, Dynamic, Derived \\
Prekop et al. (2003) [20] & Physical and logical \\
Mayrhofer (2004) [21] & Geographical, Physical, Organizational, Social, Emotional, \\
& User, Task, Action, Technological, Time \\
Wang et al. (2004) [22] & Low level, High level \\
Chen et al. (2004) [23] & Agents, time, space, events, user profiles, actions and policies. \\
Brezillon et al. (2004) [24] & Continuous, enumerative, state and descriptive context \\
Bunningen et al. (2005) [25] & Operational and conceptual \\
Chaari et al. (2005) [26] & network profile, user description/preferences, terminal \\
& characteristics, location and environment \\
Chang Xu (2005) [27] & Physical and logical \\
Razzaque et al. (2005) [28] & user, physical, network, activity, material and service context \\
Miao et al. (2006) [29] & Sensed, profiled and derived \\
& \\
&
\end{tabular}




$\begin{array}{ll}\text { Guan et al. (2007) [30] } & \text { Low level, High level } \\ \text { Chong et al. (2007) [31] } & \text { Computing, Physical, History, Identity and Time } \\ \text { Zimmerman (2007) [32] } & \text { Individuality, time, location, activity and relation } \\ \text { Miraoui et Tadj (2008) [33] } & \text { Trigger information, Quality changing information } \\ \text { Arianti Kurti (2009) [34] } & \text { User's profile, activity, location/environment } \\ \text { Soylu (2009) [35] } & \text { User and environment } \\ \text { Zhong (2009) [36] } & \text { User, System, Environment, Social, Time } \\ \text { Tamine et al. (2010) [37] } & \text { User, platform and environment. } \\ \text { Rizou et al. (2010) [38] } & \text { Low level, High level } \\ \text { Nageba E. (2011) [39] } & \text { Physical and abstract } \\ \text { Kim et al.(2012) [40] } & \text { 5W1H (Who, When, Where, What, Why and How) } \\ \text { Bin Guo (2013) [41] } & \text { Individual, social, and urban context } \\ \text { Boughareb et al. (2014) [42] } & \text { Device, task, user, document, spatio-temporal, environmental, } \\ & \text { and event } \\ \text { Ameyed (2016) [43] } & \text { Time, space and purpose. }\end{array}$

\subsection{Discussion}

Schilit et al. [6] categorized context into three conceptual entities based on three common questions: where you are that includes all information related location, common or specific names, addresses and user preferences; who you are with, the information about the people present around the user, and what resources are nearby such as machineries, smart objects, and utilities. Abowd et al. [11] introduced one of the most controversial hypotheses of defining context types. They considered location, identity of user, time, and activity as the primary context types, while they defined secondary context as the context that can be extracted using primary context. Pascoe et al. [9], Chang et al. [27], Hofer et al. [17] and Prekop et al. [20] divided the context into two main categories, the physical context that can be measured by the physical sensors and the logical context that contains abstract information about the environment or the interaction such as the user's emotional state, goals, etc. In a similar way Wang et al. [22], Guan et al. [30] and Rizou et al. [38] distinguished between two categories of context. First, we have low level or observabale context which represents the information that can be directly obtained from sensors or other sources, and secondly there is high level context or non-observable information that must be inferred from the first kind of context. Petrelli et al. [13] have seen context from a different point of view, he considered location, machine, existing platform as material context, while he identified the social aspects or relationship between individuals as part from the social context. In contrast, Gwizdka [14] used two main domains to describe the context. The internal context includes the user's state and the external context encompassing the state of the environment. Chaari [26] added more elements to the basic facets of context in specific application domains: network profile, user description/preferences, terminal characteristics, location and environment. Nageba [39] classified the components of context as physical component such as actor, organization, resource, etc. and abstract component such as process, task, service, messages, parameter, etc. Soylu et al. [35] proposed a hierarchical representation of context with two main roots is defined user and environment. Bunningen et al. [25] classified the context into two broader categories: operational and conceptual. The operational category concerns the method of 
acquisition and execution of context. On the other hand, the Conceptual category is only interested with relationships between the contexts. In the same setting, Zimmerman et al. [32] presented a formal extension for context from five fundamental categories: individuality, time, location, activity and relations. Bin Guo [41] introduced new context dimensions that involve individual, social, and urban context. Chen et al. [23] propose a four-dimensional space for context organized into active and passive context, these four dimensions are computing, physical, time and user. Klemke et al. [15] presents a simple topology for context with concentrating on the following contextual aspects: organizational, domain content based, personal and physical. Miraoui et al. [33] have organized contextual information in two classes: trigger information whose change in value causes automatic release of services provided by the pervasive system and quality changing information whose change in value causes the change of service's format. Brezillon et al. [24] proposed valuesbased categorization: continuous context, enumerative context, state context and descriptive context. Kim el al. [40] mobilizes $5 \mathrm{~W} 1 \mathrm{H}$ as the minimum information that is necessary to process physical and logical contextual information. Who (Identity), Where (Location), When (Time), What (Activity), Why and How. Boughareb et al. [42] proposes a new context taxonomy gathering what they consider all possible dimensions of context such as device, task, user, document, spatio-temporal, environmental, and event. Franklin et al. [10] illustrate their context as the person's actions. Where that action will have very different synonym depending on the situation it is used in. Antti Aaltonen et al. [18] considered the context as a group of following variables: location, target, and calendar, address book, users nearby, history, profile, direction and speed. Tamine et al. [37] modeled the context by the triplet: user, platform and environment. Henricksen [19] proposed four categories of context: sensed context includes data-acquired directly from sensors, static or permanent information that do not change over time, dynamic information that changes over time and finally derived information that can be obtained using the first three categories. As part of efforts to find a useful categorization, some authors [7, 8, $11,16,21,25,28,29]$ lists aspects of context as combinations of three, four or six elements from the composition we mentioned before.

\section{Dimension of medical context}

Like the creation of any new strategy, the process of developing the medical context should start with a clear understanding of healthcare domain. This requires going beyond all-too-common taxonomies that they don't provide practical sense of context. Structuring context information in the form of categories is a very important to organize contextual knowledge. The set of context information presented above clearly demonstrates that what is considered as context depends on what needs to be described [45]. Therefore, the proposed models discussed in the preceding section offer a general structure that should be extended to suit the medical domain. A domain specific vocabulary may raise the level of abstraction and remove ambiguity by specifying all relevant concepts. The list below outlines the basic entities that respond to requirements of medical context. 


\subsection{Person}

The persons within this healthcare environment are classified according to their functions (e.g. patients, physicians, and social). To build such medical context we need first to identify roles and responsibilities of each person, provided patients remains the concentration key point, for which all actions and decisions are directed [26]. Since patient can be characterized by their physical and psychological attributes, the person category can be subdivided into physical parameters and psychological parameters.

\subsection{Profile}

Person concept is also directly responsible for modeling the profile of the user in the medical domain. Person's profile consists of a set of basic characteristics that should be considered by a system developer to provide a suitable ubiquitous healthcare customized to the needs of the patient (e.g. Demographic, preferences). The personal profile of patient is needed, in order to automatically perform adaptations that meet the user's necessities [27].

\subsection{Time}

The medical applications, especially remote patient monitoring focuses on end-to end transmission in real time. Thus, computing systems should be aware of the time to support urgent decision or to record certain events. Usually, in telemedicine, the data measurements are used for immediate response based on real time analytics. Time, when applied to data transfer, can configure bandwidth reservation in networks, thereby enabling faster analytics, low latency for real time operation and timely delivery of critical messages [41]. Furthermore, Klein et al. [44] stressed on the importance of time synchronization for context aware applications to infer a user's situation with more accuracy because the contextual information is often aggregated from multitude of physical and virtual sensors.

\subsection{Location}

In addition to the previous contextual factors, location awareness is considered the backbones of all context aware systems [43]. With the location awareness, patients' tracking becomes easier and they can be transported to a hospital or a medical center when they need urgent intervention. This helps explain why pervasive healthcare applications require the determination of location of patient. Location often provides much deeper, more meaningful and identifiable description about the physical characteristics of the place which may have an impact on the patient's health status (e.g. the altitude harms some types of patients). There are three different ways to represent location: 1) geographic coordinate, 2) named spaces (e.g. room), and 3) relative location (e.g. describe the position of an object in relation to surrounding objects) [46]. 


\subsection{Activity}

Today, there is a growing demand of automated recognition of human activities in the health-care domain. Tracking the current situation of the users in smart space allows extending the applications with new features that may give more accurate and consistent results [48]. Furthermore, using the activity context could be useful to warn the user if they were increasing their levels of exercise in an exaggerated manner to prevent exacerbations or any serious complications.

\subsection{Technology}

This category covers any human-built thing, whether hardware or software application. This part includes computing hardware devices such as Mobile, Personal Digital Assistants (PDAs) or sensors. Technology context not only refers to computing resources but also to issues such as connectivity to a network, platform characteristics [11, 17]. In this setting, Jaydip et al. [3] refer to the importance of mobile connectivity or persistent wireless access and quality of service (e.g., the available bandwidth) to deliver healthcare service. Furthermore, these technologies cover as well the biomedical equipment used by the patients, basically there are three types of devices in the medical environment [45] : (i) fixed infrastructure equipment such as heating, ventilation and air conditioning, (ii) support equipment (e.g. microbiological sterilization and disinfection, laboratory equipment and analytical instruments) and (iii) medical equipment (e.g. vital signals monitor that includes blood pressure, cardiac monitors, respiration rate, pulse oximeters, oxygen saturation, pressure, and temperature) in addition to electrocardiogram, defibrillators, ventilators and computerized tomography.

\subsection{Environment}

The next context factor to consider when deciding to create a framework to assist healthcare provider organizations is the environment. Ubiquitous healthcare systems must recognize too, that small changes either indoor or outdoor in the environmental factors can have great impact on the patient's behavior [47]. Such a context may include humidity, temperature, pollution and pressure etc. Disease progression can be found in relation to many environmental factors [48]. For example, cold and hot weather could irritate chronic Illness symptoms and pose significant risk to chronic obstructive pulmonary disease (COPD) patient [3], also exposure to air pollutants may increase the chances of developing acute respiratory infection [46].

\subsection{Real time data}

Real time data includes signals taken by biomedical equipment or environmental sensor. Real-time data is used to control both the environmental and physical conditions. Broadly, we might take the mental status in implementation of a continuous control system by adding a set of questionnaires that they answer using a 
specified set of responses. Furthermore, this category includes a host of sites that have real time weather, air, water, and satellite information.

\subsection{History}

Historical information contains all a patient's medical history and its long-term follow-up. In practice, this part of the context should contain sufficient information about physical exam findings and prior diagnostic test results, family diseases, regular and acute medications. This parameter could help healthcare professionals to take proper decision [46]. Moreover, using historical context might improve future performance of healthcare systems. In assessing the historical context, we found that this concept (1) grants the ability for treatment to be supervised and monitored, (2) provide the possibility to exchange full health information about a patient. Therefore, that leads to a higher quality of care.

\subsection{Disease}

Disease context is presented as causal attribution that describes the relations among the symptoms, causes, and treatment [43]. Equally, to present services completely customizable according to patient status, a class of human disease is needed. This category will enrich the existing taxonomy of medical context by linking diseases with appropriate medications, consequently that would provide efficient administration for the treatment [5]. A treatment subclass is associated with multiple components such as type of treatments, condition and effect. Furthermore, disease profile may comprise a combination of severity, duration, stage, and physical feature etc. [45].

\subsection{Task}

Essentially, care providers use the context to perform task or actions and interact with patients to control precarious or suspicious situations. Hence, action is considered an essential element of the proposed categorization. According to Lasierra et al. [5] medical tasks will comprise four different types: monitoring task, analysis task, planning task and execution task. The relation between these tasks is governed by a set of conditions expressed using rules.

\subsection{Event}

Likewise, event can be part of task, but we prefer to define it as an independent concept to detect expected events when occur. To put it more simply, we could say that such concept is very necessary because of the nature of the remote health care system, which takes as input the physical attributes that indicate the existing conditions to make the analysis of more complex incidents, e.g. exacerbation. 


\subsection{Organization}

Healthcare organization is a generic term refers to any healthcare entity (hospital, clinic, etc.). Organization is responsible for the management and delivery of healthcare services, by making decision, performing processes and using resources. For example, (1) define and monitor the delivery process of the service care, (2) assign a care provider and a medical equipment when required for a patient, (3) manage and allocate human and physical resources between services and (4) manage collaboration with other centers [5].

\subsection{Policies}

Context categorization provides a conceptual framework for understanding of context at the application-level. Therefore, this context can be further used at application-level for management of policies. Such as policies include security, integrity, confidentiality and availability, as well as end patient's privacy. Due to the sensitive nature of this information, we believe that is important to satisfy strict privacy and security requirements. The security refers to guarantee reliable message transmission where all security systems should be equipped with mechanisms that can be used to resolve error and carrying information via well-protected channels. Likewise, privacy of information is necessary to establish policies for user and resource to assign permissions such who owns health information, and how restricted is access to it [47].

\section{Case Study: medical context of COPD}

To motivate the use of our model, we introduce in the following an example of context as a pattern of chronic disease. Context of COPD is designed to provide personalized care plans for patients to identify symptoms, risk factors, and effective self-management of COPD. The graphical representation below (Fig. 1) contains the domain-specific context of COPD. These domain-specific components can be interchangeably compatible with any kind of chronic diseases. The context elements listed in this cluster is an example of a domain-specific context consisting of 51 context elements related to COPD. We must mention here that this domain specific context information is not intended to provide an exhaustive representation of the context in the COPD domain, it is simply to show the context elements for the domain as identified in our content analysis of context. Practically, COPD could be a relevant example when designing tele-monitoring system in the healthcare domain. This implementation is used to identify to which extent current taxonomy approach can generate telemedicine services adapted to the current contextual needs of patients. The structure of the COPD context comprise group of 14 entities used for analyzing such specific health problem. In this work, we created a custom context based on fourteen types of real world concepts that intelligent system should consider when reasoning over COPD context. Thus, Fig. 1 provides a clearer and deeper view of COPD domain. The figure reflects the way we read and our perception and understanding of typical hierarchical structure of the context categories. 


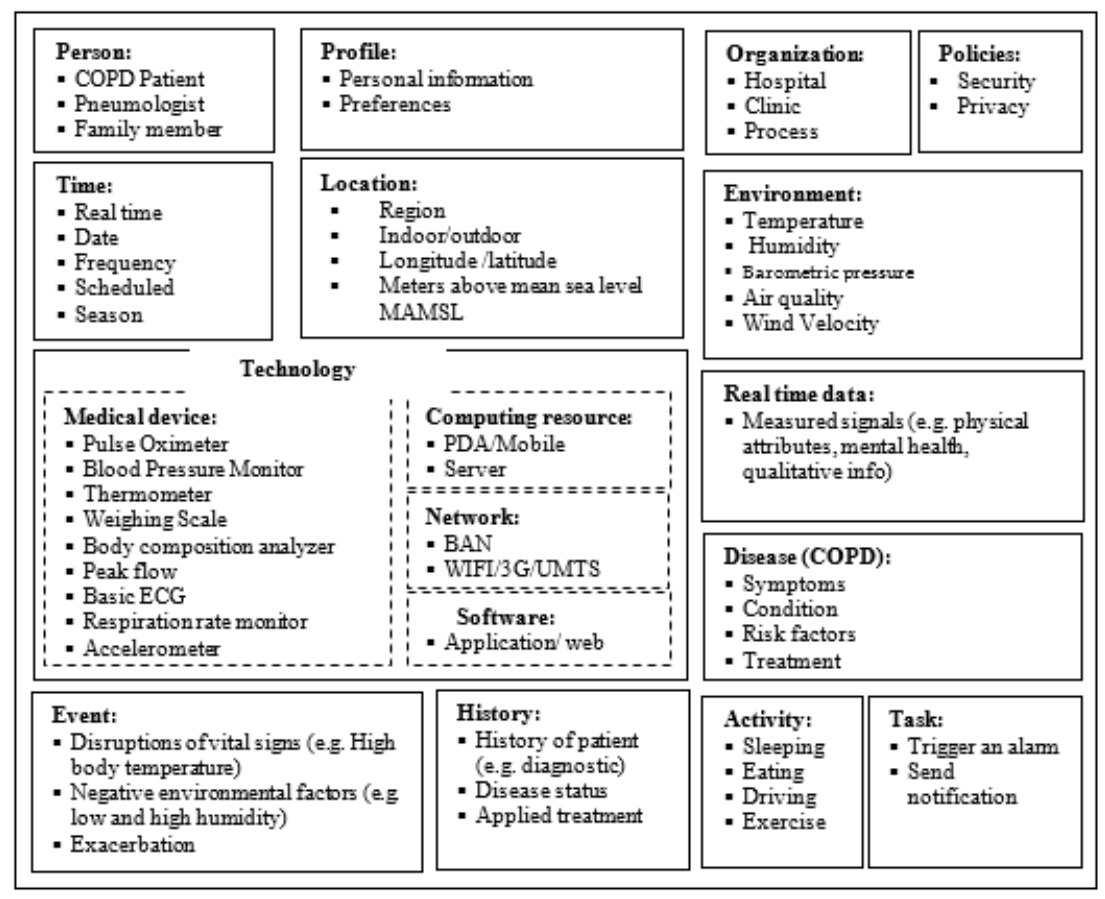

Fig. 1. Domain specific context for COPD

Each of these categories has several subcategories that retains to itself of a list of internal context elements. Person context contains the subcategories patient which can be seen as an axial centric point of the medical context, besides physician and family member. Profile context includes personal information about COPD patient and his care providers. Time context may comprise current time to intervene in a timely manner as well as terms related to date and seasons. Location context could be outdoor and indoor place such as home, office, park etc. Technology context is the pervasive network of technologies that surrounds people, added to it the medical devices for monitoring human vital signs, such as body temperature, heart rate, respiration rate, blood pressure, pulse oxygenation, and blood glucose. Environmental context contains all factors that affects COPD patients such as temperature, humidity, barometric pressure, air quality and wind Velocity. Activity context is ranging from everyday home activities (e.g. eating, sleeping) to driving or traveling. Event context is very important to detect abnormal situations specifically all that is related to exacerbation. Task context could be useful to describe monitoring process, trigger events or provide recommendations. Disease context includes symptoms, characteristics, risk factors and treatment of COPD. History is quite essential to register health profile of patient such as diagnosis, lab tests, scans in addition to the applied therapy. Real time data consists of the instantaneous data e.g. physical attributes, mental health. Policies context establish the privacy and security requirements which is considered one of the most important pillars of medical 
services both traditional and modern. These benchmarks are valid to any type of illnesses, since only the internal contents of the main context parameters need to be changed.

\section{Conclusion}

Since real world entities and their relationships are very essential for describing events and situations, identifying a comprehensive list of medical context becomes an important matter to support the healthcare system. In this paper, we conducted a detailed analysis to determine the different dimensions of context. This work dissects the medical domain into a set of concepts that can be used as general terms for the design of healthcare systems. Recent research has identified location, environment, time, person and activity as the most crucial parameters for describing real-world things. Our taxonomy of medical context relies on fourteen components that besides the previous parameters, contains also other items such as the task, history, data, event, disease, organization and policies. This new categorization would be a guideline and an effective strategy that may help developers to find their context structure. The organization of context content is an important issue to consider during creating context awareness system in healthcare environments. This study provides further contribution to the field of ubiquitous computing. For future work we will continue to explore and investigate how the idea of context categorization can provide more features for context awareness system.

\section{References}

1. Arianit Kurti. "Exploring the multiple dimensions of context:" PHD thesis, Växjö University Press, 2009.

2. Gaëtan Rey, Joëlle Coutaz. The Contextor Infrastructure for Context-Aware Computing. Engineering HCI research group, CLIPS IMAG, Grenoble, France June 2004.

3. Jaydip Sen, Arijit Ukil, A QoS-aware end-to-end connectivity management algorithm for mobile applications, Proceedings of the Third Annual ACM Bangalore Conference, p.1-9, January 22-23, 2010, Bangalore, India.

4. Dey, A., Abowd, G., and Salber D., "A conceptual framework and a toolkit for supporting the rapid prototyping of context-aware applications. Human-Computer Interaction”, 2000.

5. N. Lasierra, “A three stage ontology-driven solution to provide personalized care to chronic patients at home”. Journal of biomedical informatics, vol. 46, 2013, pp 516-52.

6. Schilit, B. Disseminating Active Map Information to Mobile Hosts. IEEE Network, 8(5). 1994.

7. Ryan, N. Enhanced Reality Fieldwork: the Context-Aware Applications in Archaeology (1997) 23.

8. Hull, R., Neaves, P., Bedford-Roberts, J.Towards Situated Computing. 1st International Symposium on Wearable Computers (1997) 146-153.

9. Pascoe, J. Adding Generic Contextual Capabilities to Wearable Computers. 2nd International Symposium on Wearable Computers (1998) 92-99. 
10. Franklin, D. Spring Symposium on Intelligent Environments, Technical Report SS-98-02 (1998) 155-160.

11. Dey, A.K., Abowd, G.D., Wood, A. CyberDesk: A Framework for Providing SelfIntegrating Context-Aware Services. Knowledge-Based Systems, 11 (1999) 3-13.

12. Chen, G. A survey of context-aware mobile computing research (Vol. 1, No. 2.1, pp. 2-1). Technical Report TR2000-381,, Dartmouth College (2000).

13. D. Petrelli, E. “Modeling Context is Like Taking Pictures.”CHI2000 Workshop, 2000.

14. J.Gwizdka. "What's in the Context."CHI2000 Workshop (2000).

15. Klemke, R. Context Framework. In Proceedings of the International Conference on Practical Aspects of Knowledge Management - (Oct. 30-31, Basel, Switzerland). 2000.

16. Gross, T. and Specht M. Awareness in context-aware information systems. Tagungsband der 1. Fachübergreifenden Konferenz "Mensch \& Computer", Bonn, Germany (2001).

17. Hofer, T." Context-awareness on mobile devices - the hydrogen approach," Proceedings of the 36th Annual Hawaii International Conference on System Sciences, pp.292-302. (2002).

18. Aaltonen, Antti. "A Context Visualization Model for Wearable Computers.” SEMWEB (2002).

19. Henricksen, K. A Framework for Context-Aware Pervasive Computing Applications. Ph.D. Thesis, University of Queensland, Queensland, Queensland. 2003.

20. Prekop, P., \& Burnett, M. Activities, context and ubiquitous computing. Computer Communications, 26(11), 1168-1176 (2003).

21. Mayrhofer, R. “An Architecture for Context Prediction”. PhD thesis, Johannes Kepler University of Linz, Austria, October 2004.

22. Wang, X.H., J.S. Dong, C.Y. Chin and S.R. Hettiarachchi, 2004. Semantic Space: An Infrastructure for Smart Spaces. IEEE Pervasive Computing, 3 (2): 32-39, July-Sept. 2004.

23. Chen H. A. "An Ontology for Context-Aware Pervasive Computing Environments”, vol. 18, num. 3, 2004, p. 197-207, Cambridge University Press.

24. Brézillon, P. Learning and explanation in a context-based representation: Application to incident solving on subway lines. 2004.

25. Van Bunningen, A. H., Feng, L., \& Apers, P. M. (2005, April). Context for ubiquitous data management. In Ubiquitous Data Management, 2005. UDM 2005. International Workshop on IEEE (pp. 17-24).

26. Chaari, T., Laforest, F., \& Celantano, A.. Design of context-aware applications based on web services. Technical report, LIRIS UMR 5205 CNRS/INSA de Lyon/Université Claude Bernard. (2004).

27. Chen F. J., Warden A. C., \& Chang H. T. Motivators that do not motivate: The case of Chinese EFL learners and the influence of culture on motivation. TESOL Quarterly, 39(4), 609-33. (2005).

28. Razzaque, M.A., Dobson,S., \& Nixon,P. Categorization and modeling of quality in context information. In Proceedings of th IJCAI 2005. Workshop on AI and autonomic communications. Edinburgh. Scotland.

29. Y. Miao, X. Tao, Z. Shen, Z. Liu, and C. Miao. The equivalence ofcognitive map, fuzzy cognitive map and multi value fuzzy cognitivemap. In IEEE International Conference on Fuzzy Systems, 2006.

30. Guan, J., Xiang, P., McBride, R., \& Bruene, A. Achievement goals, social goals and students' reported persistence and effort in high school PE. 2006 Journal of Teaching in PE, 25, 58-74.

31. S. K. Chong. "Contextaware sensors and data muling," in Context awareness for selfmanaging systems (devices, applications and networks) proceeding. Berlin : VDEVerlag, 2007, pp. 103-117.

32. Zimmerman, A., Lorenz, A. \& Oppermann, R., 2007. An Operational Definition of Context. In: Lecture Notes in Computer Science. s.l.:Springer-Verlag Berlin, pp. 558- 571. 
33. M. Miraoui, C. Tadj, and C. b. Amar, "Architectural survey of context-aware systems in pervasive computing environment," Ubiquitous Computing and Communication Journal, vol. 3, 2008.

34. Kurti, A. Exploring the multiple dimensions of context: Implications for the design and development of innovative technology-enhanced learning environments. Stockholm University, May 28, 2009.

35. Soylu, A.; De Causmaecker1, P. \& Desmet, P. (2009). Context and Adaptivity in Pervasive Engineering, Journal of Software, Vol.4, No.9, (November, 2009), pp.992-1013, ISSN 1796-217X.

36. G. Jun-zhong, “Context aware computing,” Journal of East China Normal University (Natural Science), vol. 5, pp. 1-20, 2009.

37. Tamine-Lechani L, Boughanem M, Daoud M (2010) Evaluation of contextual information retrieval effectiveness: overview of issues and research. Knowl Inf Syst 24(1):1-34

38. S. Rizou. "A system for distributed context reasoning," in Autonomic and Autonomous Systems (ICAS), 2010 Sixth International Conference on, march 2010, pp. 84 -89.

39. Nageba, E. A Model Driven Ontology-based Architecture for Supporting the Quality of Services in Pervasive Telemedicine Applications. Proceedings the 3rd International Conference on Pervasive Computing Technologies for Healthcare, London UK, IEEE Computer Society, pp. 1-8, Apr 2009

40. S. Kim. Smart learning services based on smart cloud computing. Sensors 11(8), 78357850 (2011)

41. Bin Guo. the Internet of Things to Embedded Intelligence. World Wide Web 16, 4 (2013), 399-420.

42. D. Boughareb., Context in information retrieval, International Conference on Control, Decision and Information Technologies (CoDIT), 3-5 Nov. 2014, France.

43. Darine AMEYED. Modélisation et spécification formelle de contexte et sa prédiction dans les systèmes diffus . PHD thesis. L’École de technologie supérieure (ETS) 2016.

44. Bernd Niklas Klein. On the importance of time synchronization for context aware applications.PHD thesis, Kassel University Press 2011.

45. J. Bardram, Applications of Context-Aware Computing in Hospital Work, In Proceedings of 2004 ACM Symposium on Applied Computing, pp. 1574 - 1579, ACM Press, 2004.

46. Levandoski, J., Sarwat, M., Eldawy, A., Mokbel, M.: LARS: a location-aware recommender system. In: ICDE, pp. 450-461 (2012)

47. X. Xu, J. Tang, X. Zhang, X. Liu, H. Zhang, and Y. Qiu, "Exploring techniques for vision based human activity recognition: Methods, systems, and evaluation," Sensors, vol. 13, no. 2, pp. 1635-1650, 2013.

48. Ajami Hicham, Mcheick Hamid et Elkhaled Zayan. Survey of health care context models : prototyping of healthcare context framework. Dans : Summer Simulation Multi-Conference (SummerSim'16) , July 24-27, 2016, Montreal, Quebec. 